\title{
$\mathrm{H}_{2}$ production pathways
}

\section{in nutrient-replete mixotrophic}

Chlamydomonas cultures under low light. Response to the commentary article "On the pathways feeding the $\mathrm{H}_{2}$ production process in nutrient-replete, hypoxic conditions," by Alberto Scoma and Szilvia Z. Tóth

\author{
David González-Ballester ${ }^{*}$ (0, Jose Luis Jurado-Oller, Aurora Galván, Emilio Fernández and Alexandra Dubini
}

\begin{abstract}
Background: A recent Commentary article entitled "On the pathways feeding the $\mathrm{H}_{2}$ production process in nutrientreplete, hypoxic conditions" by Dr. Scoma and Dr. Tóth, Biotechnology for Biofuels (2017), opened a very interesting debate about the $\mathrm{H}_{2}$ production photosynthetic-linked pathways occurring in Chlamydomonas cultures grown in acetate-containing media and incubated under hypoxia/anoxia conditions. This Commentary article mainly focused on the results of our previous article "Low oxygen levels contribute to improve photohydrogen production in mixotrophic non-stressed Chlamydomonas cultures," by Jurado-Oller et al., Biotechnology for Biofuels (7, 2015; 8:149).

Main body: Here, we review some previous knowledge about the $\mathrm{H}_{2}$ production pathways linked to photosynthesis in Chlamydomonas, especially focusing on the role of the PSII-dependent and -independent pathways in acetatecontaining nutrient-replete cultures. The potential contributions of these pathways to $\mathrm{H}_{2}$ production under anoxia/ hypoxia are discussed.
\end{abstract}

Conclusion: Despite the fact that the PSII inhibitor DCMU is broadly used to discern between the two different photosynthetic pathways operating under $\mathrm{H}_{2}$ production conditions, its use may lead to distinctive conclusions depending on the growth conditions. The different potential sources of reductive power needed for the PSII-independent $\mathrm{H}_{2}$ production in mixotrophic nutrient-replete cultures are a matter of debate and conclusive evidences are still missing.

Keywords: Chlamydomonas, Hydrogen, DCMU, Acetate, Algae, Biofuels, Biomass, Low light, Oxygen

\section{Background}

The alga Chlamydomonas reinhardtii (Chlamydomonas throughout) is able to perform $\mathrm{H}_{2}$ photoproduction, as well as fermentative $\mathrm{H}_{2}$ production. Two distinct pathways

\footnotetext{
*Correspondence: q62gobad@uco.es

Departamento de Bioquímica y Biología Molecular, Facultad de Ciencias,

Universidad de Córdoba, Campus de Rabanales, Edif. Severo Ochoa, 14071 Córdoba, Spain
}

have been described to explain $\mathrm{H}_{2}$ photoproduction. One of them, termed as direct pathway or PSII-dependent pathway, requires the participation of the entire photosynthetic electron chain, including PSII and PSI. Electrons originated from the water photolysis at the level of the PSII reach the PSI where they are transferred to the ferredoxin 1 (FDX1) and are finally donated to the primary hydrogenase of Chlamydomonas, HYDA1. The necessary participation of the PSII in this pathway implies that $\mathrm{O}_{2}$ is produced 
simultaneously with $\mathrm{H}_{2}$. Since hydrogenases are very sensitive to $\mathrm{O}_{2}$, under regular growth conditions, this process is very transitory, and $\mathrm{H}_{2}$ production quickly ceases as $\mathrm{O}_{2}$ accumulates. This pathway might have an important physiological role during the dark to light transitions of the cells. An alternative pathway termed as indirect pathway or PSIIindependent pathway can also lead to $\mathrm{H}_{2}$ photoproduction. In this case, electrons do not originate from water photolysis at the PSII level but from a non-photosynthetic reduction of the plastoquinone (PQ) pool. It has been shown that a plastoquinone-reducing type II $\mathrm{NAD}(\mathrm{P}) \mathrm{H}$ dehydrogenase (NDA2) plays a crucial role in this process. In this pathway, electrons flow from the PQ pool to the PSI, and similarly to the PSII-dependent pathway; they are finally donated to the HYDA1 via FDX1. Note that this pathway does not require the participation of the PSII, and no $\mathrm{O}_{2}$ is produced. Finally, Chlamydomonas is also able to produce $\mathrm{H}_{2}$ linked to fermentative pathways. In this case, electrons are donated to HYDA1 from the activity of a Pyruvate Ferredoxin Reductase (PFR), which catalyzes the oxidation of pyruvate to acetyl-CoA under anoxic conditions. PFR activity is coupled to FDX1, which accepts the electrons from the catalyzed reaction and donates them to HYDA1. It has been shown that PFR is also able to use, in addition to pyruvate, oxaloacetate as a substrate (reviewed in [1]).

Chlamydomonas is able to uptake acetate and uses it as a carbon source. Mixotrophic and autotrophic growth conditions refer to the presence or absence of acetate, respectively. Importantly, most studies about $\mathrm{H}_{2}$ production in Chlamydomonas have been done using TrisAcetate-Phosphate (TAP) medium, and it is well known that acetate strongly enhances $\mathrm{H}_{2}$ production in Chlamydomonas (reviewed in [1]).

The present Commentary Article is a response to the Commentary Article written by Scoma and Toth (Biotechnology for Biofuels, 2017). Here, we will discuss the few available data about the photosynthetic $\mathrm{H}_{2}$ production in mixotrophic nutrient-replete cultures incubated under low light conditions [2,3], particularly the role of the PSIIdependent and -independent pathways. Moreover, we will discuss and try to clarify the available literature concerning the contribution of the PSII-independent pathway to $\mathrm{H}_{2}$ production, in both mixotrophic nutrient-deplete and -replete medium, when using the PSII inhibitor DCMU.

\section{Main text}

Comparison of the recent data obtained for $\mathrm{H}_{2}$ production in TAP cultures incubated under low light: commentaries on the publications of Scoma et al. [2], Jurado-Oller et al. [3], and responses to the Commentary Article of Scoma and Toth (2017)

The commentary article published by Scoma and Toth (Biotechnology for Biofuels 2017) discussed the pathways feeding the $\mathrm{H}_{2}$ production process in nutrient-replete Chlamydomonas cultures incubated under low light, and highlighted the different results and data interpretation obtained by Scoma et al. [2] and Jurado-Oller et al. [3].

These two publications studied $\mathrm{H}_{2}$ production in Chlamydomonas mixotrophic cultures incubated under low light during several days; 25 days in the case of Scoma et al. [2] and 10 days in Jurado-Oller et al. [3]. The former report used an illumination of $20 \mu \mathrm{mol}$ photons $\mathrm{m}^{-2} \mathrm{~s}^{-1}$, while the later used a range of light intensities (from 12 to $50 \mu \mathrm{mol}$ photons $\mathrm{m}^{-2} \mathrm{~s}^{-1}$ ). For comparative reasons, we will refer here to the data from Jurado-Oller et al. [3] obtained under $12 \mu \mathrm{mol}$ photons $\mathrm{m}^{-2} \mathrm{~s}^{-1}$ since this is the condition that they examined the most. Note that, some other important differences between both publications' experimental set-ups could explain some discrepancies. Scoma et al. [2] used a D1 mutant, twice more concentration of acetate than the standard formulation of the TAP media, purged cultures, an initial cell concentration of $40-80 \mathrm{mg}$ chl. $\mathrm{L}^{-1}$, and a ratio gas/liquid of 1.4 in the bioreactors. While Jurado-Oller et al. [3] used a strain without any photosynthetic phenotype (704), a standard TAP media recipe, no purged cultures, an initial cell concentration of $10 \mathrm{mg}$ chl. $\mathrm{L}^{-1}$, and a gas/liquid ratio of 0.4 in the bioreactors. All these factors can greatly influence $\mathrm{H}_{2}$ production patterns.

Scoma et al. [2] reported that sealed cultures were able to produce some $\mathrm{H}_{2}$ after $4 \mathrm{~h}\left(\approx 9-11 \mathrm{ml} \mathrm{l}^{-1}\right)$, and afterwards no more $\mathrm{H}_{2}$ production was observed during the next 10 days (this 10 days period is termed as Phase 1 by these authors). AQThese data are in good agreement with those published by Jurado-Oller et al. [3], who reported $\mathrm{H}_{2}$ production in sealed cultures after $24 \mathrm{~h}\left(\approx 7 \mathrm{ml} \mathrm{l}^{-1}\right)$. Both studies reported that $\mathrm{H}_{2}$ production lasted less than $24 \mathrm{~h}$, and afterwards no more $\mathrm{H}_{2}$ production was observed during the next 10 days. In the report of Jurado-Oller et al. [3], the headspaces of the bioreactors were not purged and atmospheric oxygen concentrations were present at the beginning of the experiment indicating that in the presence of acetate, low light-incubated cultures can quickly reach anoxia and produce $\mathrm{H}_{2}$ very fast. The earlier time at which $\mathrm{H}_{2}$ production was observed in Scoma et al. [2], relative to Jurado-Oller et al. [3] ( 4 vs 24 h), could be reflecting the fact that the earlier report used purged cultures and high cell concentration cultures, which allow a faster establishment of anaerobiosis.

Very interestingly, Scoma et al. [2] observed a second $\mathrm{H}_{2}$ production phase (Phase 2) not observed by JuradoOller et al. [3]. This $\mathrm{H}_{2}$ production phase occurred after $250 \mathrm{~h}$ of the establishment of the hypoxic conditions (around 10 days), and was sustained for about 14 days. Authors describe the $\mathrm{H}_{2}$ production observed during 
Phase 1 as "traces of $\mathrm{H}_{2}$ " and production during Phase 2 as a "sharp $\mathrm{H}_{2}$ accumulation." However, these differences in the $\mathrm{H}_{2}$ production rates should be considered more carefully. According to some of the published data ([2]; Figure 4), the $\mathrm{H}_{2}$ accumulation level about $125 \mathrm{~h}$ after the beginning of Phase 2 is lower than the initial $\mathrm{H}_{2}$ accumulation observed during the first $25 \mathrm{~h}$ of Phase 1 . Unfortunately, the experiments performed by Jurado-Oller et al. [3] were stopped after 10 days, which is the precise time where Scoma et al. [2] observed their Phase 2 of $\mathrm{H}_{2}$ production, making impossible any comparison between these two reports in this regard.

Importantly, while Jurado-Oller et al. [3] concluded that $\mathrm{H}_{2}$ production is mostly linked to the PSII-independent pathway, Scoma et al. [2] concluded that $\mathrm{H}_{2}$ production during Phase 2 is primordially due to the PSII-dependent pathway. However, the report published by Scoma et al. [2] does not provide any conclusive data supporting this statement. Indirectly, Scoma et al. [2] based this affirmation on three facts: (1) starch accumulation is prevented in the cultures; (2) $\mathrm{H}_{2} / \mathrm{CO}_{2}$ ratios were lower in the presence of DCMU; (3) Fv/Fm values decreased during $\mathrm{H}_{2}$ production. However, under our understanding, none of these facts demonstrates that $\mathrm{H}_{2}$ production in these cultures is linked to the PSII activity. Moreover, a decrease of $\mathrm{Fv} / \mathrm{Fm}$ during $\mathrm{H}_{2}$ production is not incompatible with a PSII-independent production. Interestingly, they reported almost no inhibition of the $\mathrm{H}_{2}$ production when adding DCMU ([2]; Figure $4 \mathrm{~b}$ and Table 2), which is indicative of a dominant PSII-independent $\mathrm{H}_{2}$ production. Data represented in Figure 4b [2] illustrate how $\mathrm{H}_{2}$ is being produced in the presence of DCMU at the same time and to the same extent as the control cultures. Strikingly, authors increased light intensity in cultures containing DCMU (but not in control cultures) at the same time as Phase 2 started, which makes the interpretation of the data difficult.

On the other hand, Jurado-Oller et al. [3] stated that $\mathrm{H}_{2}$ production is mostly PSII-independent in aerated cultures since cultures treated with DCMU were able to produce up to $81 \%$ of $\mathrm{H}_{2}$ relative to control cultures (aeration was performed every $24 \mathrm{~h}$ by deliberately opening the bioreactors in a sterile atmosphere). Note that in the report of Jurado-Oller et al. [3], control cultures incubated in dark showed a substantially lower $\mathrm{H}_{2}$ production than cultures incubated in low light, indicating a minor role for the fermentative $\mathrm{H}_{2}$ production pathway operating during low light conditions. As explained in more detail below, the contribution of the PSII-independent pathway (deduced from the DCMU treatments) could be underestimated when oxygen availability of the cultures is very limited. Different operating conditions can enhance (or limit) PSII-independent vs -dependent $\mathrm{H}_{2}$ production, but undoubtedly, the $\mathrm{H}_{2}$ production observed in aerated cultures by Jurado-Oller et al. [3] is mainly linked to a PSII-independent pathway.

In Scoma et al. [2] and in the commentary article of Scoma and Toth (2017, Biotechnology for Biofuels), authors stated that under their experimental conditions, acclimation to hypoxia of the cultures required up to 10 days.

This concept of "acclimation" could be subjective and not easy to understand, since authors were able to detect $\mathrm{H}_{2}$ production after $4 \mathrm{~h}$, implying that hypoxia was already established. Moreover, one might expect that cells incubated for up to 25 days $(600 \mathrm{~h})$ under hypoxic conditions could have their viability very compromised. In this regard, it is interesting to note that according to Figure 2 of Scoma et al. [2], oxygen is accumulated over 25 days in the headspace of the cultures incubated under 20 PAR (initial cell concentration of $40 \mathrm{mg}$ chl. $\mathrm{L}^{-1}$ ), which could explain why cells were able to survive for such a long period. On the other side, Jurado-Oller et al. [3] reported that cultures containing four times less cells and incubated under 12 and 22 PAR showed no oxygen accumulation over 10 days (Figure 1b, in Jurado-Oller et al. [3]); moreover, the cell viability of these cultures was very compromised after this period (data not shown).

Finally, both publications reported that the starch reserves are not mobilized during $\mathrm{H}_{2}$ production, unlike in S-depleted cultures. On the contrary, the starch reserves increased during $\mathrm{H}_{2}$ production indicating that $\mathrm{H}_{2}$ production under these conditions is not linked to the mobilization of the starch reserves. Scoma et al. [2] reported a decrease in the protein content concomitant with the $\mathrm{H}_{2}$ production, which could potentially support PSII-independent production. On the other hand, Jurado-Oller et al. [3] suggested that acetate assimilation/dissimilation may be linked (directly or indirectly) to the PSII-independent $\mathrm{H}_{2}$ production during these conditions. This suggestion is based on the correlation of acetate uptake and $\mathrm{H}_{2}$ production when using DCMU (see below for a more detailed explanation). Although $\mathrm{H}_{2}$ production rates are not directly proportional to the acetate uptake rates, the former is impaired when the latter is also severely impaired. A tentative metabolic model is proposed by Jurado-Oller et al. [3], trying to describe how acetate assimilation/dissimilation can contribute to $\mathrm{H}_{2}$ production, linking acetate uptake with the TCA and glyoxylate cycles and with a non-photochemical reduction of the photosynthetic electron chain. A similar model was previously proposed by others [4]. However, these models are lacking any experimental evidence so far. 
The contribution of the PSII-dependent and -independent pathways to $\mathrm{H}_{2}$ production in the presence of acetate The PSII inhibitor DCMU is broadly used to determine the respective contribution of the two $\mathrm{H}_{2}$ photoproduction pathways present in Chlamydomonas. If DCMU is added to cultures under conditions promoting $\mathrm{H}_{2}$ production, an inhibition of the $\mathrm{H}_{2}$ production is observed. This rate of inhibition is used to determine the contribution of the PSII-dependent and -independent pathways. Through this approach, several publications have evaluated the contribution of the PSII-dependent pathway in mixotrophic nutrient-depleted [5-9] and -replete cultures $[2,3,10,11]$. Interestingly, very different values have been assigned, in both nutrient-replete and -depleted medium, to the contribution of the PSII-independent pathway, ranging from 0 to $100 \%$ of the total $\mathrm{H}_{2}$ production (Table 1). The contribution of the PSII-independent pathway has been largely studied in S-depleted cultures, and it is generally assumed that this pathway contributes to about $10-20 \%$ of the total $\mathrm{H}_{2}$ production in this medium $[7,9,12]$. However, it has been reported that several parameters greatly affect the PSII-independent contribution in S-depleted cultures when using $\operatorname{DCMU}[7,8,13]$. Two parameters seem to be crucial for the differences in $\mathrm{H}_{2}$ production in these cultures: (1) the time at which DCMU is added to the cultures (right after $\mathrm{S}$ depletion or few days after $\mathrm{S}$ depletion) $[7,8,13]$; and (2) the cell density of the cultures [8]. The major contribution of the PSII-independent pathway is obtained when low cell density cultures are used or when DCMU is added few days after $\mathrm{S}$ depletion.

Similarly, Jurado-Oller et al. [3] also reported that in mixotrophic nutrient-replete cultures incubated in low light, the addition of DCMU caused very different effects on $\mathrm{H}_{2}$ production depending on the growth conditions. In this case, oxygen availability of the cultures (provided by aeration) greatly increased the contribution of the PSII-independent pathway on $\mathrm{H}_{2}$ production (81\%) when compared to non-aerated cultures (21\%) or purged cultures $(36 \%)$. Recently, in a commentary article of Scoma and Toth (Biotechnology for Biofuel 2017), these data were considered as contradictory, but under our point of view, they are reflecting how cultures under different physiological conditions respond differently to a PSII inhibitor, similarly to what has been reported in S-depleted media. From these data, JuradoOller et al. [3] proposed that the effect of DCMU on $\mathrm{H}_{2}$ production in mixotrophic cultures incubated under low light is modulated by the presence of oxygen in the cultures; the more the oxygen the less the inhibition. In contrast to the inhibition caused by DCMU observed by Jurado-Oller et al. [3] in non-aerated cultures $(79 \%$ inhibition of the total $\mathrm{H}_{2}$ production), Scoma et al. [2] observed very little inhibition of $\mathrm{H}_{2}$ production when using DCMU in sealed cultures (14\% inhibition of the total $\mathrm{H}_{2}$ production according to the data presented in Table 2, [2]). Interestingly, Jurado-Oller et al. [3] described no oxygen accumulation in the headspaces of non-aerated cultures incubated under 12 PAR (Figure $1 \mathrm{~b}$ in Jurado-Oller et al. [3]); however, Scoma et al. [2] reported the presence of oxygen in the headspaces of the cultures (Figure 2, [2]).

Since PSII activity is dispensable to obtain $81 \%$ of total $\mathrm{H}_{2}$ production in aerated cultures incubated under low light, Jurado-Oller et al. [3] proposed that the PSIIindependent pathway is the primordial via to produce $\mathrm{H}_{2}$ in these cultures. Moreover, Jurado-Oller et al. [3] also demonstrated that acetate uptake is greatly dependent on oxygen availability. Hence, the effect of DCMU can also affect greatly the acetate uptake rates depending on the different oxygenation regimes of the cultures. Cultures supplemented with DCMU under aeration regime were essentially unaffected in their capacity to uptake acetate (relative to control cultures), whereas non-aerated and purged cultures presented a severe impairment of the acetate uptake. Based on these facts (acetate uptake and $\mathrm{H}_{2}$ production in the presence of DCMU), Jurado-Oller et al. [3] suggested that the $\mathrm{H}_{2}$ production in nutrientreplete cultures under low light could be linked (directly or indirectly) to the acetate uptake rates. Note that, as deduced from purged cultures, the releasing of the $\mathrm{H}_{2}$ partial pressure (without providing oxygen) in the presence of DCMU is not greatly contributing in increasing neither the contribution of the PSII-independent $\mathrm{H}_{2}$ production (36\%) nor the acetate uptake rates (acetate uptake is essentially blocked) (Figure 3 in Jurado-Oller et al. [3]). This is indicating that oxygen availability, and not the releasing of the $\mathrm{H}_{2}$ partial pressure, is affecting these two processes. Overall, the data provided by Jurado-Oller et al. [3] revealed that the inhibitory effect caused by DCMU on $\mathrm{H}_{2}$ production in mixotrophic cultures incubated under low light could not be entirely linked to the lack of electrons provided by the PSII activity, but mainly to an indirect effect related to oxygen availability.

The data provided by Jurado-Oller et al. [3] and their interpretation could be in partial agreement with the data reported for S-depleted cultures. The different effects of DCMU on $\mathrm{H}_{2}$ production in S-depleted cultures were explained based on the capacity of the cultures to accumulate starch before the addition of DCMU, since this inhibitor blocks also starch accumulation [7]. Starch is accumulated during the oxygenic phase and constitutes the main source of reductants feeding the PSII-independent $\mathrm{H}_{2}$ production once the cultures reach the anoxic phase. Authors proposed that starch accumulation before 
Table 1 Comparison of the in vivo PSII-independent contribution to $\mathrm{H}_{2}$ production under different conditions

\begin{tabular}{|c|c|c|c|c|c|c|c|}
\hline Reference & $\begin{array}{l}\text { In vivo PSII-independ- } \\
\text { ent contribution }{ }^{a}(\%)\end{array}$ & Media & Strain & Cell density ${ }^{b}$ & $\begin{array}{l}\text { Purged } \\
\text { cultures }\end{array}$ & PAR $^{c}$ & Notes \\
\hline \multirow[t]{2}{*}{ Healey [11] } & 100 & $N R$ & $\begin{array}{l}\text { C. moewusii } \\
\text { ICC } 97\end{array}$ & & & 300 (lux) & Dark-light cycle adaptation \\
\hline & 100 & $N R$ & $\begin{array}{l}\text { C. dysosmos } \\
\text { ICC } 242\end{array}$ & & & 400 (lux) & Dark-light cycle adaptation \\
\hline Gibbs et al. [10] & 18 & $N R$ & F60 & & Yes & $100 \mathrm{~W} / \mathrm{m}^{2}$ & $\begin{array}{l}\text { Dark adaptation ( } 2 \mathrm{~h} \text { ) } \\
\text { DCMU and acetate added simultane- } \\
\text { ously } \\
\text { Acetate uptake is inhibited by } 91 \% \text { in } \\
\text { the presence of DCMU }\end{array}$ \\
\hline Scoma et al. [2] & $86^{d}$ & $N R$ & $\begin{array}{l}\text { L159I-N230Y } \\
\text { (D1 mutant) }\end{array}$ & 80 & Yes & 100 & $\begin{array}{l}\text { DCMU added after } 150 \mathrm{~h} \\
2 \mathrm{X} \text { acetate in TAP formulation } \\
\text { In cultures without DCMU, } \mathrm{O}_{2} \text { accumu- } \\
\text { lated in the headspaces }\end{array}$ \\
\hline \multirow[t]{3}{*}{$\begin{array}{l}\text { Jurado-Oller } \\
\text { et al. [3] }\end{array}$} & 21 & $N R$ & 704 & 10 & No & 12 & $\begin{array}{l}\text { DCMU added at } 0 \mathrm{~h} \\
\text { Atmospheric } \mathrm{O}_{2} \text { level when DCMU is } \\
\text { added } \\
\mathrm{O}_{2} \text { levels remained very low after } 24 \mathrm{~h}\end{array}$ \\
\hline & 36 & $N R$ & 704 & 10 & Yes & 12 & DCMU added at $0 \mathrm{~h}$ \\
\hline & 81 & $N R$ & 704 & 10 & No & 12 & $\begin{array}{l}\text { DCMU added at } 0 \mathrm{~h} \\
\text { Atmospheric } \mathrm{O}_{2} \text { level when DCMU is } \\
\text { added } \\
\text { Cultures aerated every } 24 \mathrm{~h}\end{array}$ \\
\hline \multirow{5}{*}{$\begin{array}{l}\text { Hemschemeier } \\
\text { et al. [8] }\end{array}$} & 0 & $-S$ & cc124 & 20 & No & 100 & DCMU immediately after S depletion \\
\hline & 40 & $-S$ & cc124 & 20 & No & 100 & DCMU added $17 \mathrm{~h}$ after $\mathrm{S}$ depletion \\
\hline & $80-65$ & $-S$ & cc124 & 20 & No & 100 & $\begin{array}{l}\text { DCMU added during } \mathrm{H}_{2} \text { production } \\
\text { phase } \\
\mathrm{H}_{2} \text { measured by MIMS }\end{array}$ \\
\hline & 40 & $-S$ & cc124 & 27 & No & 100 & $\begin{array}{l}\text { DCMU added during } \mathrm{H}_{2} \text { production } \\
\text { phase } \\
\mathrm{H}_{2} \text { measured by MIMS }\end{array}$ \\
\hline & 100 & $-S$ & cc124 & 17 & No & 100 & $\begin{array}{l}\text { DCMU added during } \mathrm{H}_{2} \text { production } \\
\text { phase } \\
\mathrm{H}_{2} \text { measured by MIMS }\end{array}$ \\
\hline \multirow[t]{2}{*}{$\begin{array}{l}\text { Fouchard et al. } \\
\text { [7] }\end{array}$} & $\approx 0$ & $-S$ & cc124 & $5 \times 10^{6} \mathrm{cells} / \mathrm{ml}$ & Yes & 110 & $\begin{array}{l}\text { DCMU immediately after } S \text { depletion } \\
\mathrm{O}_{2} \text { levels near } 0 \% \text { when DCMU is added }\end{array}$ \\
\hline & 20 & $-S$ & cc124 & $5 \times 10^{6} \mathrm{cells} / \mathrm{ml}$ & Yes & 110 & $\begin{array}{l}\text { DCMU was added } 24 \mathrm{~h} \text { after } \mathrm{S} \text { depletion } \\
\mathrm{O}_{2} \text { levels near } 10 \% \text { when DCMU is added }\end{array}$ \\
\hline \multirow[t]{3}{*}{$\begin{array}{l}\text { Laurinavichene } \\
\text { et al. [13] }\end{array}$} & 51 & $-S$ & cc124 & $20-28$ & Yes & 30 & $\begin{array}{l}\text { DCMU added after } 46 \text { h of } S \text { depletion } \\
\mathrm{H}_{2} \text { production is maximal as this time }\end{array}$ \\
\hline & 32 & $-S$ & cc124 & $20-28$ & Yes & 30 & DCMU added after $70 \mathrm{~h}$ of $\mathrm{S}$ depletion \\
\hline & 28 & $-S$ & cc124 & $20-28$ & Yes & 30 & DCMU added after $94 \mathrm{~h}$ of $\mathrm{S}$ depletion \\
\hline $\begin{array}{l}\text { Chochois et al. } \\
\text { [12] }\end{array}$ & 10 & $-S$ & 330 & $4 \times 10^{6} \mathrm{cells} / \mathrm{ml}$ & Yes & 200 & DCMU added $24 \mathrm{~h}$ after $\mathrm{S}$ depletion \\
\hline Antalet al. [15] & 30 & $-S$ & cc124 & $4 \times 10^{6}$ cells $/ \mathrm{ml}$ & Yes & 25 & \\
\hline Philips et al. [5] & 100 & $-\mathrm{N}$ & cc124 & $5 \times 10^{6} \mathrm{cells} / \mathrm{ml}$ & No & 60 & DCMU added $72 \mathrm{~h}$ after $\mathrm{N}$ depletion \\
\hline $\begin{array}{l}\text { Volgusheva et al. } \\
\text { [6] }\end{array}$ & 26 & $-M g$ & cc124 & 7 & No & 80 & $\begin{array}{l}\text { DCMU added } 8 \text { days after Mg depletion } \\
\mathrm{O}_{2} \text { levels near } 0 \text { when DCMU is added }\end{array}$ \\
\hline
\end{tabular}

NR Nutrient Replete

a Measured as the $\%$ of $\mathrm{H}_{2}$ production in the presence of DCMU (relative to control cultures)

b In $\mathrm{mg}$ chl. $\mathrm{L}^{-1}$ unless otherwise indicated

c Photosynthetic active radiation (PAR) in $\mu$ mol photons $\mathrm{m}^{-2} \mathrm{~s}^{-1}$, unless otherwise indicated

d Data according to Table 2 in original publication 
the $\mathrm{H}_{2}$ production phase is not impaired if the addition of DCMU takes place few days after $\mathrm{S}$ depletion or if low cell density cultures are used [7, 8]. Under these conditions, the contribution of the PSII-independent pathway is more significant. Cell culture density could influence effective light intensity and thereby PSII activity. Thus, authors proposed that the PSII activity is essential to accumulate starch during the aerobic phase, which in turn will contribute to the PSII-independent $\mathrm{H}_{2}$ production. However, an alternative possibility cannot be ruled out, which is not based on the PSII activity per se but on the oxygen availability and acetate uptake rates. Oxygen availability could allow acetate uptake, which in turn will also impact starch accumulation.

Note that those conditions favoring PSII-independent production in S-depleted cultures are precisely conditions where oxygen availability could be higher. On the contrary, severe anoxic conditions can lead to very low acetate uptake, low starch levels, and very low PSII-independent $\mathrm{H}_{2}$ production. In general, there is a good correlation in the literature between the oxygen availability of the cultures and the degree of contribution of the PSIIindependent pathway (Table 1) in both nutrient-replete and -deplete cultures. Conditions favoring anoxia such as purged cultures, high cell concentration, early addition of DCMU in S-depleted cultures, or different mutations causing low $\mathrm{O}_{2}$ evolution can result in an underestimation of the PSII-independent pathway.

\section{Conclusions}

Incubation of mixotrophic nutrient-replete cultures under restricted light conditions lead to a rapid $(<24 \mathrm{~h})$ accumulation of $\mathrm{H}_{2}[2,3]$. Interestingly, these cultures can show a second $\mathrm{H}_{2}$ production phase after prolonged incubation ( $>10$ days) under hypoxic conditions [2]. This strategy could be an alternative way to produce $\mathrm{H}_{2}$ to those based on nutrient-depleted conditions with the additional advantage of producing biomass simultaneously $[2,3]$. Unfortunately, compared with the production in S-depleted cultures, $\mathrm{H}_{2}$ production under this condition is lower, which makes even more difficult any potential biotechnological application. Still, the number of publications studying $\mathrm{H}_{2}$ production under mixotrophic nutrient-replete cultures is very scarce and more knowledge could be gained in the future. In addition, in mixotrophic low light cultures, the release of the $\mathrm{H}_{2}$ pressure and/or the supplementation with additional acetate can lead to a substantial optimization of the $\mathrm{H}_{2}$ production [3].

It has been proposed that in mixotrophic S-depleted cultures, the activity of the PSII during the aerobic phase is indispensable for $\mathrm{H}_{2}$ production [7,8]. Yet, as demonstrated by Jurado-Oller et al. [3], the PSII activity is dispensable for $\mathrm{H}_{2}$ production in mixotrophic nutrientreplete cultures incubated under low light and under aeration. However, under nutrient-replete conditions, the relative contributions of the PSII-dependent and -independent pathways are still a matter of debate since their contribution may vary with the culture conditions. Further studies will be necessary to demonstrate conclusively the relative contribution of these two pathways in this condition. In any case, the starch reserves do not seem to be linked to $\mathrm{H}_{2}$ production [2, 3, 14]. Catabolism of proteins $[2,14]$ or acetate assimilation/dissimilation [3, $4,10]$ are potential processes that can provide reductive power for PSII-independent $\mathrm{H}_{2}$ production. However, conclusive results to characterizing the main electron source used for PSII-independent $\mathrm{H}_{2}$ production under this condition are still missing.

In the available literature, the use of DCMU to calculate the contribution of the two photosynthetic $\mathrm{H}_{2}$ production pathways can generate a large variety of effects, depending on the specific growth conditions. It is possible that oxygen levels in the cultures can determine the degree of inhibition caused by DCMU [3]. The effect caused by DCMU could not only be linked to the inhibition of the PSII activity per se and to the concomitant loss of electrons that can enter in the photosynthetic chain, but also to the elimination of the main source of oxygen in sealed cultures. Oxygen is indispensable for the uptake of acetate [3], which in turn can also impair starch accumulation. Moreover, the lack of oxygen can also have an important impact under illumination conditions on chlororespiration, photorespiration, and the Mehler reaction. All these factors could be linked to (or influence) $\mathrm{H}_{2}$ production in Chlamydomonas under different conditions, which make difficult the interpretation of the results regarding the PSII-independent contribution to $\mathrm{H}_{2}$ production when using DCMU. In any case, the PSII-independent contribution could be greatly underestimated when adding DCMU to cultures where oxygen is severely depleted or when starch accumulation is prevented in the case of S-depleted cultures.

\section{Authors' contributions}

DG-B analyzed the results, interpreted the data, and wrote the manuscript. $A D$ significantly contributed to the analysis and interpretation of the data, and in the writing of the manuscript. EF and AG supervised and coordinated the study, and helped in the writing of the manuscript. All authors read and approved the final manuscript.

\section{Competing interests}

All authors declare no competing interests.

\section{Funding}

This work was funded by the MINECO (Ministerio de Economia y Competitividad, Spain, Grant No. BFU2015-70649-P [granted to E.F. and A.G.]), supported by the European "Fondo Europeo de Desarrollo Regional (FEDER)" program, the Plan E program (CONV 188/09 [granted to EF]), the Ramon y Cajal program 
(RYC-2011-07671 [granted to D.G-B.]), the Talent Hub program (291780, granted to A.D.), Junta de Andalucía grants (P12-BIO-502 and BIO-128 [granted to A.G. and E.F.], and "Plan Propio de la Universidad de Córdoba."

\section{Publisher's Note}

Springer Nature remains neutral with regard to jurisdictional claims in published maps and institutional affiliations.

Received: 15 February 2017 Accepted: 22 April 2017

Published online: 05 May 2017

\section{References}

1. Gonzalez-Ballester D, Luis Jurado-Oller J, Fernandez E. Relevance of nutrient media composition for hydrogen production in Chlamydomonas. Photosynth Res. 2015;125:395-406.

2. Scoma A, Durante L, Bertin L, Fava F. Acclimation to hypoxia in Chlamydomonas reinhardtii: can biophotolysis be the major trigger for longterm $\mathrm{H}_{2}$ production? New Phytol. 2014;204:890-900.

3. Jurado-Oller JL, Dubini A, Galván A, Fernández E, González-Ballester D. Low oxygen levels contribute to improve photohydrogen production in mixotrophic non-stressed Chlamydomonas cultures. Biotechnol Biofuels. 2015;8:149.

4. Willeford KO, Gibbs M. Localization of the Enzymes Involved in the Photoevolution of $\mathrm{H}(2)$ from Acetate in Chlamydomonas reinhardtii. Plant Physiol. 1989;90:788-91.

5. Philipps G, Happe T, Hemschemeier A. Nitrogen deprivation results in photosynthetic hydrogen production in Chlamydomonas reinhardtii. Planta. 2012;235:729-45.
6. Volgusheva A, Kukarskikh G, Krendeleva T, Rubina A, Mamedov F. Hydrogen photoproduction in green algae Chlamydomonas reinhardtii under magnesium deprivation. RSC Adv. 2014;5:5633-7.

7. Fouchard S, Hemschemeier A, Caruana A, Pruvost J, Legrand J, Happe T, Peltier G, Cournac L. Autotrophic and mixotrophic hydrogen photoproduction in sulfur-deprived Chlamydomonas cells. Appl Environ Microbiol. 2005;71:6199-205.

8. Hemschemeier A, Fouchard S, Cournac L, Peltier G, Happe T. Hydrogen production by Chlamydomonas reinhardtii: an elaborate interplay of electron sources and sinks. Planta. 2008;227:397-407.

9. Kosourov S, Seibert M, Ghirardi ML. Effects of extracellular pH on the metabolic pathways in sulfur-deprived, $\mathrm{H}_{2}$-producing Chlamydomonas reinhardtii cultures. Plant Cell Physiol. 2003;44:146-55.

10. Gibbs M, Gfeller RP, Chen C. Fermentative Metabolism of Chlamydomonas reinhardii: III. Photoassimilation of Acetate. Plant Physiol. 1986;82:160-6.

11. Healey FP. Hydrogen evolution by several algae. Planta. 1970;91:220-6.

12. Chochois V, Dauvillée D, Beyly A, Tolleter D, Cuiné S, Timpano H, Ball S, Cournac L, Peltier G. Hydrogen production in Chlamydomonas: photosys tem II-dependent and -independent pathways differ in their requirement for starch metabolism. Plant Physiol. 2009;151:631-40.

13. Laurinavichene T, Tolstygina I, Tsygankov A. The effect of light intensity on hydrogen production by sulfur-deprived Chlamydomonas reinhardtii. J Biotechnol. 2004;114:143-51.

14. Degrenne B, Pruvost J, Legrand J. Effect of prolonged hypoxia in autotrophic conditions in the hydrogen production by the green microalga Chlamydomonas reinhardtii in photobioreactor. Bioresour Technol. 2011;102:1035-43.

15. Antal T, Volgusheva A, Kukarskih G, Krendeleva T, Rubin A. Relationships between $\mathrm{H}-2$ photoproduction and different electron transport pathways in sulfur-deprived Chlamydomonas reinhardtii. Int J Hydrogen Energy. 2009:34:9087-94

\section{Submit your next manuscript to BioMed Central and we will help you at every step:}

- We accept pre-submission inquiries

- Our selector tool helps you to find the most relevant journal

- We provide round the clock customer support

- Convenient online submission

- Thorough peer review

- Inclusion in PubMed and all major indexing services

- Maximum visibility for your research

Submit your manuscript at www.biomedcentral.com/submit 